\title{
Dynamics of the Zeraoulia-Sprott map revisited
}

\author{
G. Chen, E.V. Kudryashova, N.V. Kuznetsov, G.A. Leonov \\ City University of Honk Hong, China \\ Saint-Petersburg State University, Russia \\ University of Jyväskylä, Finland \\ Institute for Problems in Mechanical Engineering of RAS, Russia
}

\section{Introduction.}

In the paper [13], a 1-D map related to the dynamics of some evolutionary processes (see, e.g. recent surveys on evolutionary algorithms and their applications to the study of chaotic dynamics [14, 15]) was investigated. Later, its extended two-dimensional version was studied in [3]:

$$
\left(\begin{array}{l}
x \\
y
\end{array}\right) \mapsto\left(\begin{array}{c}
\frac{-a x}{1+y^{2}} \\
x+b y
\end{array}\right)
$$

where $a$ and $b$ are bifurcation parameters. In the paper "Some Open Problems in Chaos Theory and Dynamics" [4, the following problem was formulated: Find regions in the $a-b$ plane in which the map (1) is bounded and chaotic in the rigorous mathematical definition of chaos and boundedness of attractors.

In the present paper, the boundedness of the attractors of the map (1) is studied and the corresponding analytical estimation of absorbing set is obtained, thereby giving an answer to the above problem.

Introduce the notation

$$
h\left(y_{k}\right)=\frac{-a}{1+y_{k}^{2}}, \quad k \in \mathbb{N}_{0} .
$$

The eigenvalues and eigenvectors of the map are defined by the following relation:

$$
\begin{gathered}
\left(\begin{array}{l}
x_{k+1} \\
y_{k+1}
\end{array}\right)=A\left(\begin{array}{l}
x_{k} \\
y_{k}
\end{array}\right)+\left(\begin{array}{c}
\frac{a x_{k} y_{k}^{2}}{1+y_{k}{ }^{2}} \\
0
\end{array}\right), \quad A=\left(\begin{array}{cc}
-a & 0 \\
1 & b
\end{array}\right), \\
\operatorname{det}(A-p I)=\operatorname{det}\left(\begin{array}{cc}
-a-p & 0 \\
1 & b-p
\end{array}\right)=(-a-p)(b-p)=p^{2}+p(a-b)-a b .
\end{gathered}
$$

Thus, the eigenvalues are

$$
p=b, \quad p=-a \text {. }
$$

For the eigenvalue $p=-a$, one has

$$
\left(\begin{array}{cc}
-a & 0 \\
1 & b
\end{array}\right)\left(\begin{array}{l}
x \\
y
\end{array}\right)=\left(\begin{array}{l}
-a x \\
-a y
\end{array}\right)
$$

Hence, the corresponding eigenvectors are proportional to

$$
\left(\begin{array}{c}
1 \\
-\frac{1}{a+b}
\end{array}\right) \text {. }
$$


For the eigenvalue $p=b$, one has

$$
\left(\begin{array}{cc}
-a & 0 \\
1 & b
\end{array}\right)\left(\begin{array}{l}
x \\
y
\end{array}\right)=\left(\begin{array}{l}
b x \\
b y
\end{array}\right)
$$

and, thus, $x=0$.

Next, the following 4 cases are considered, with the associated properties proved:

$|a|<1,|b|<1$ : Global asymptotic stability;

$|a|<1,|b|>1$ : Existence of unbounded solutions;

$|a|>1,|b|<1$ : Localization of nontrivial global attractor;

$|a|>1,|b|>1$ : Existence of unbounded solutions.

The cases of $|a|=1$ or $|b|=1$ are not considered in this paper, which require special consideration therefore will be discussed elsewhere.

\section{Case 1: $|a|<1,|b|<1$. Global asymptotic stability.}

There exists $\delta>0$ such that $\max (|a|,|b|)<\delta<1$. Then, $\left|h\left(y_{k}\right)\right|<\delta<1, \forall k$, and

$$
\left|x_{k}\right| \leq \delta^{k}\left|x_{0}\right|, \quad \forall k \geq 0
$$

Since $\delta<1$, one has

$$
x_{k} \underset{k \rightarrow \infty}{\longrightarrow} 0
$$

For $y_{k}$, one has

$$
\begin{aligned}
\left|y_{k+1}\right| & =\left|b y_{k}+x_{k}\right|=\left|b^{2} y_{k-1}+b x_{k-1}+x_{k}\right| \\
& \leq|b|^{k+1}\left|y_{0}\right|+|b|^{k}\left|x_{0}\right|+|b|^{k-1}\left|x_{1}\right|+|b|^{k-2}\left|x_{2}\right|+\cdots+|b|\left|x_{k-1}\right|+\left|x_{k}\right| \\
& \leq|b|^{k+1}\left|y_{0}\right|+(k+1) \delta^{k}\left|x_{0}\right| .
\end{aligned}
$$

Taking into account of the fact that $|b|<\delta<1$, one gets $y_{k+1} \underset{k \rightarrow \infty}{\longrightarrow} 0$. Thus, for $|a|<1,|b|<1$, the global asymptotic stability is confirmed (see Fig. 11).
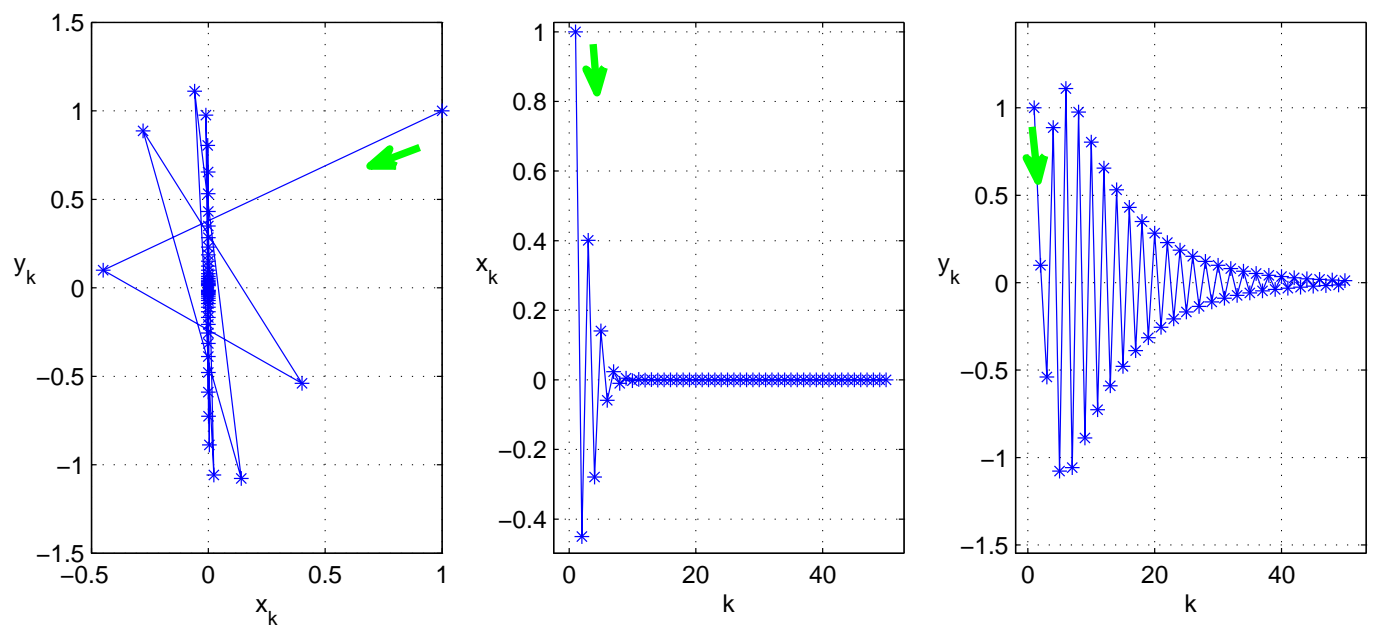

Figure 1: Case $1(|a|<1,|b|<1): \quad a=0.9, \quad b=-0.9, \quad x_{1}=1, \quad y_{1}=1, \quad k=[1,50]$. 


\section{Case 2: $|a|<1,|b|>1$. The existence of unbounded solutions.}

It will be shown that if $|a|<1,|b|>1$, then there exists $\left(x_{0}, y_{0}\right)$ such that

$$
\left|x_{k}\left(x_{0}, y_{0}\right)\right| \underset{k \rightarrow \infty}{\longrightarrow} 0, \quad\left|y_{k}\left(x_{0}, y_{0}\right)\right| \underset{k \rightarrow \infty}{\longrightarrow} \infty
$$

For $|a|<1$, there exists $\delta>0$ such that $|a|<\delta<1$. Then, $\left|h\left(y_{k}\right)\right|<\delta<1, \forall k \geq 0$, and

$$
\left|x_{k}\right| \leq \delta^{k}\left|x_{0}\right|, \quad \forall k \geq 0
$$

For $y_{k}$, one has

$$
\begin{aligned}
y_{k+1} & =b y_{k}+x_{k}=b^{2} y_{k-1}+b x_{k-1}+x_{k} \\
& =b^{k+1} y_{0}+b^{k} x_{0}+b^{k-1} x_{1}+b^{k-2} x_{2}+\cdots+b x_{k-1}+x_{k} \\
& =b^{k+1} y_{0}+b^{k}\left(x_{0}+\frac{x_{1}}{b}+\frac{x_{2}}{b^{2}}+\cdots+\frac{x_{k-1}}{b^{k-1}}+\frac{x_{k}}{b^{k}}\right) .
\end{aligned}
$$

Taking into account the fact that $\left|x_{k}\right| \leq \delta^{k}\left|x_{0}\right|$, where $|a|<\delta<1$, one has

$$
\begin{aligned}
\left|y_{k+1}\right| & \geq|b|^{k+1}\left|y_{0}\right|-|b|^{k}\left(\left|x_{0}\right|+\frac{\delta^{1}\left|x_{0}\right|}{|b|}+\frac{\delta^{2}\left|x_{0}\right|}{|b|^{2}}+\cdots+\frac{\delta^{k-1}\left|x_{0}\right|}{|b|^{k-1}}+\frac{\delta^{k}\left|x_{0}\right|}{|b|^{k}}\right) \\
& \geq b^{k+1}\left|y_{0}\right|-b^{k}\left(\left|x_{0}\right|+\frac{\delta^{1}\left|x_{0}\right|}{|b|}+\frac{\delta^{2}\left|x_{0}\right|}{|b|^{2}}+\cdots+\frac{\delta^{k-1}\left|x_{0}\right|}{|b|^{k-1}}+\frac{\delta^{k}\left|x_{0}\right|}{|b|^{k}}\right) .
\end{aligned}
$$

For the sum of the geometric (infinitely decreasing) series, one has

$$
S_{k}\left(\left|x_{0}\right|, \frac{\delta}{|b|}\right)=\left|x_{0}\right|+\frac{\delta^{1}\left|x_{0}\right|}{|b|}+\frac{\delta^{2}\left|x_{0}\right|}{|b|^{2}}+\cdots+\frac{\delta^{k-1}\left|x_{0}\right|}{|b|^{k-1}}+\frac{\delta^{k}\left|x_{0}\right|}{|b|^{k}} \leq \frac{\left|x_{0}\right|}{1-\frac{\delta}{|b|}}=\frac{|b|\left|x_{0}\right|}{|b|-\delta} .
$$

Therefore,

$$
\left|y_{k+1}\right| \geq|b|^{k+1}\left|y_{0}\right|-|b|^{k}\left(S_{k}\left(\left|x_{0}\right|, \frac{\delta}{|b|}\right)\right) \geq|b|^{k+1}\left|y_{0}\right|-|b|^{k+1} \frac{\left|x_{0}\right|}{|b|-\delta} \geq|b|^{k+1}\left(\left|y_{0}\right|-\frac{\left|x_{0}\right|}{|b|-1}\right) .
$$

If $\left|y_{0}\right| \geq \frac{\left|x_{0}\right|}{|b|-1}$, then $\left|y_{k+1}\left(x_{0}, y_{0}\right)\right| \underset{k \rightarrow \infty}{\longrightarrow} \infty$ (see Figs. 2 and 3 .
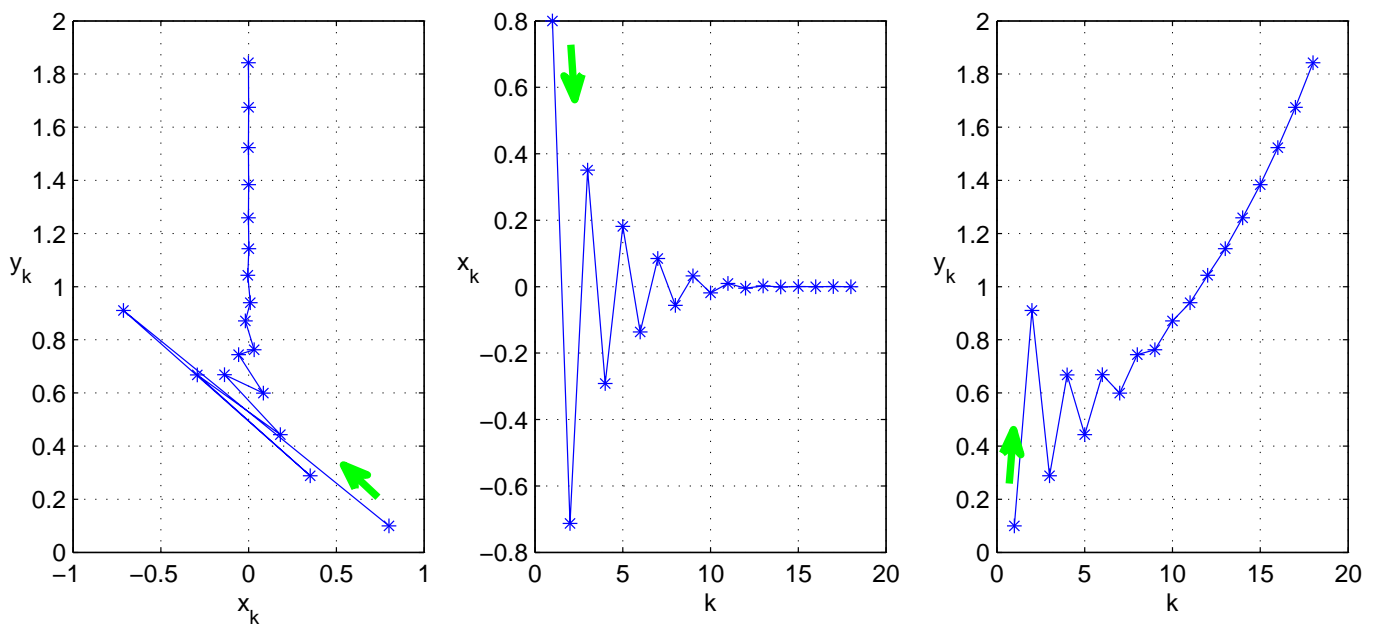

Figure 2: Case 2a $(|a|<1,|b|>1, b>0): \quad a=0.9, \quad b=1.1, \quad x_{1}=0.8, \quad y_{1}=0.1, \quad k=[1,18]$. 

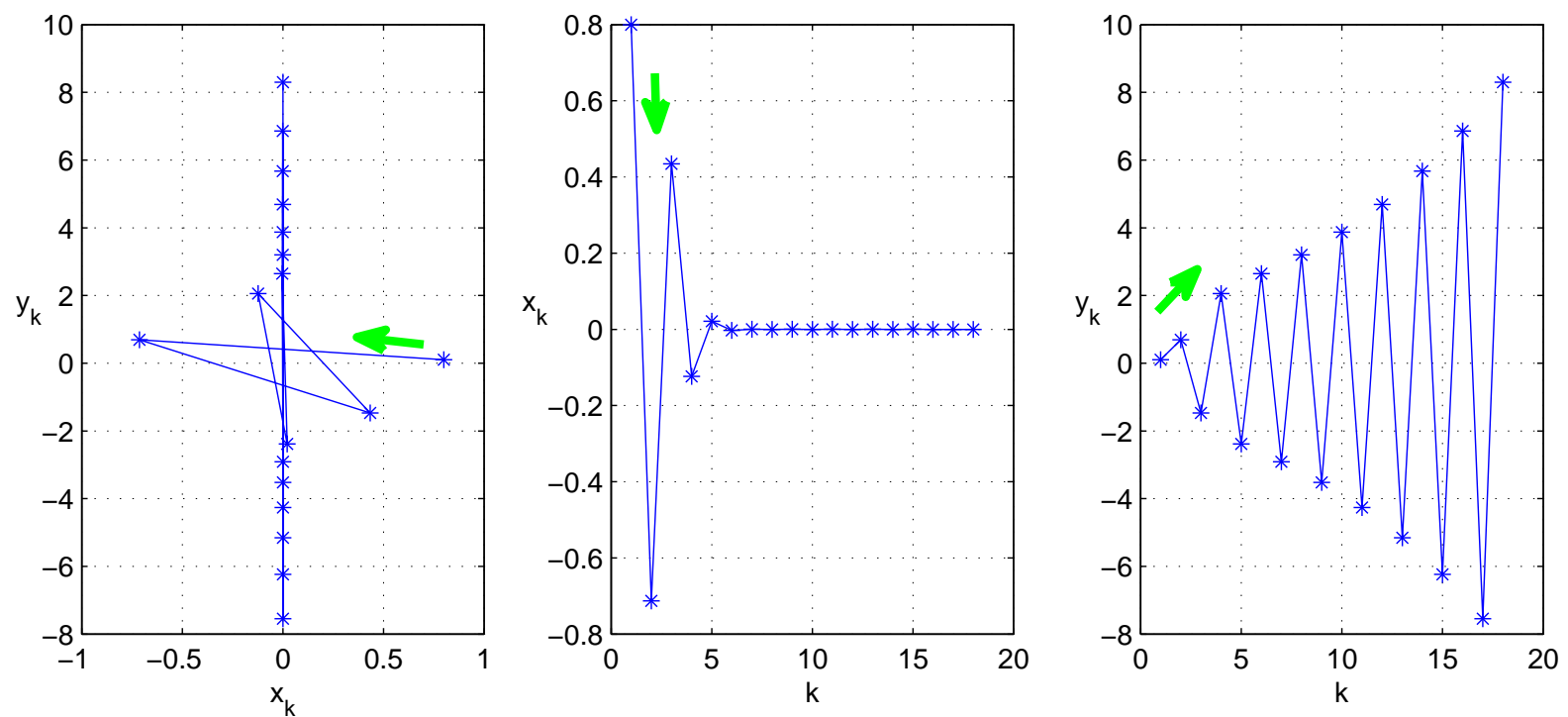

Figure 3: Case 2b $(|a|<1,|b|>1, b<0): \quad a=0.9, \quad b=-1.1, \quad x_{1}=0.8, \quad y_{1}=0.1, \quad k=[1,18]$.

\section{Case 3: $|a|>1,|b|<1$. The localization of global attractor.}

Let $0<\delta<1$ and introduce the notations

$$
R_{y}=\sqrt{|a|-1+\delta|a|}, \quad R_{x}=|b| R_{y}+\sqrt{a^{2}-1+\delta a^{2}} .
$$

Lemma 1. If $|a|>1$ and $|b|<1$, then for any $\left|x_{0}\right|$ and $\left|y_{0}\right|>R_{y}$, one has

$$
\left|x_{1}\right|<\left|x_{0}\right| \frac{1}{1+\delta}
$$

Proof. Since

$$
\begin{aligned}
\left|x_{0}\right|-\left|x_{1}\right| & =\left|x_{0}\right|\left(1-\frac{|a|}{1+y_{0}^{2}}\right) \geq\left|x_{0}\right|\left(1-\frac{|a|}{1+R_{y}^{2}}\right) \\
& =\left|x_{0}\right|\left(1-\frac{|a|}{1+|a|-1+\delta|a|}\right)=\left|x_{0}\right|\left(1-\frac{1}{1+\delta}\right),
\end{aligned}
$$

one has

$$
\left|x_{1}\right| \leq\left|x_{0}\right| \frac{1}{1+\delta}
$$

Lemma 2. If $|a|>1$ and $|b|<1$, then for $\left|y_{0}\right| \leq R_{y}$ and $\left|x_{0}\right|>R_{x}$, one has

$$
\left|x_{2}\right| \leq\left|x_{0}\right| \frac{1}{(1+\delta)}<\left|x_{0}\right|
$$

Proof. For $y_{1}$, one has

$\left|y_{1}\right|=\left|x_{0}+b y_{0}\right| \geq\left|x_{0}\right|-\left|b y_{0}\right| \geq\left|x_{0}\right|-|b| R_{y}>R_{x}-|b| R_{y}=|b| R_{y}+\sqrt{a^{2}-1+\delta a^{2}}-|b| R_{y}=\sqrt{a^{2}-1+\delta a^{2}}$.

Therefore,

$$
\frac{a^{2}}{1+y_{1}^{2}}<\frac{a^{2}}{1+a^{2}-1+\delta a^{2}}=\frac{1}{1+\delta}<1
$$


and

$$
\left|x_{0}\right|-\left|x_{2}\right|=\left|x_{0}\right|-\frac{a^{2}\left|x_{0}\right|}{\left(1+y_{0}^{2}\right)\left(1+y_{1}^{2}\right)}=\left|x_{0}\right|\left(1-\frac{1}{\left(1+y_{0}^{2}\right)} \frac{a^{2}}{\left(1+y_{1}^{2}\right)}\right) \geq\left|x_{0}\right|\left(1-\frac{1}{1+\delta}\right) .
$$

Consequently, $\left|x_{2}\right| \leq\left|x_{0}\right| \frac{1}{1+\delta}<\left|x_{0}\right|$.

By Lemma 1 and Lemma 2, one gets the following results.

Corollary 1. For any $x_{0}, y_{0}$, there exists $n \in \mathbb{N}_{0}$ such that

$$
\left|x_{n}\right| \leq R_{x}
$$

Corollary 2. If $\left|x_{0}\right| \leq R_{x}$, then $\left|x_{m}\right| \leq a^{2} R_{x}, \forall m \geq 0$.

Proof. Let $\left|x_{1}\right|>R_{x}$. If $\left|y_{1}\right|>R_{y}$, then $\left|x_{2}\right| \leq\left|x_{1}\right|$. If $\left|y_{1}\right| \leq R_{y}$, then $\left|x_{3}\right|<\left|x_{1}\right|$. Therefore,

$$
\left|x_{m}\right| \leq \max \left(\left|x_{1}\right|,\left|x_{2}\right|\right) \leq\left|a x_{1}\right| \leq a^{2}\left|x_{0}\right| \leq a^{2} R_{x}, \quad \forall m>0
$$

Lemma 3. If $|a|>1$ and $|b|<1$, then for $\left|x_{0}\right| \leq M$ and $\left|y_{0}\right|>\frac{M+\delta}{1-|b|}$, where $M>0$ and $\delta>0$, one has

$$
\left|y_{1}\right|<\left|y_{0}\right|-\delta<\left|y_{0}\right|
$$

Proof. Since

$$
\left|y_{0}\right|-\left|y_{1}\right|=\left|y_{0}\right|-\left|b y_{0}+x_{0}\right| \geq\left|y_{0}\right|-|b|\left|y_{0}\right|-\left|x_{0}\right| \geq\left|y_{0}\right|(1-|b|)-\left|x_{0}\right|>\frac{M+\delta}{1-|b|}(1-|b|)-M=\delta
$$

one has

$$
\left|y_{1}\right|<\left|y_{0}\right|-\delta<\left|y_{0}\right|
$$

Corollary 3. For $\left|x_{0}\right| \leq R_{x}$ and $\left|y_{0}\right|>\frac{a^{2} R_{x}+\delta}{1-|b|}$, there exists $n \in \mathbb{N}_{0}$ such that

$$
\left|x_{n}\right| \leq a^{2} R_{x}, \quad\left|y_{n}\right| \leq \frac{a^{2} R_{x}+\delta}{1-|b|}
$$

Proof. By Corollary 2, one has $\left|x_{n}\right| \leq a^{2} R_{x}, \forall n \geq 0$. By Lemma 3, there exists $n$ such that $\left|y_{n}\right| \leq \frac{a^{2} R_{x}+\delta}{1-|b|}$.

Lemma 4. If $|a|>1$ and $|b|<1$, then for $\left|x_{0}\right| \leq M$ and $\left|y_{0}\right| \leq \frac{M+\delta}{1-|b|}$, where $M>0$ and $\delta>0$, one has

$$
\left|y_{1}\right|<\frac{M+\delta}{1-|b|}
$$

Proof.

$$
\left|y_{1}\right|=\left|b y_{0}+x_{0}\right| \leq|b|\left|y_{0}\right|+\left|x_{0}\right| \leq|b| \frac{M+\delta}{1-|b|}+M=\frac{M+|b| \delta}{1-|b|}<\frac{M+\delta}{1-|b|} .
$$


Corollary 4. If $\left|x_{0}\right| \leq R_{x}$, then there exists $N>0$ such that

$$
\left|y_{n}\right| \leq \frac{a^{2} R_{x}+\delta}{1-|b|}, \quad \forall n>N
$$

Proof. By Corollaries 2 and 3 , there exists $n>0$ such that

$$
\left|x_{n}\right| \leq a^{2} R_{x}, \quad\left|y_{n}\right| \leq \frac{a^{2} R_{x}+\delta}{1-|b|},
$$

where $\left|x_{m}\right| \leq a^{2} R_{x}, \forall m \geq 0$. It follows from Lemma 4 with $M=a^{2} R_{x}$ that

$$
\forall k>n, \quad\left|y_{k}\right| \leq \frac{a^{2} R_{x}+\delta}{1-|b|}
$$

Therefore, by Corollaries 1,2 and 4 for any $x_{0} \neq 0, y_{0} \neq 0$, there exists $n>0$ such that, $\forall k>n$,

$$
\begin{aligned}
& \left|x_{k}\right| \leq a^{2}\left(|b| \sqrt{|a|-1+|a| \delta}+\sqrt{a^{2}-1+a^{2} \delta}\right)=a^{2} R_{x} \\
& \left|y_{k}\right| \leq \frac{a^{2}\left(|b| \sqrt{|a|-1+|a| \delta}+\sqrt{a^{2}-1+a^{2} \delta}\right)+\delta}{1-|b|}=\frac{a^{2} R_{x}+\delta}{1-|b|} .
\end{aligned}
$$

Thus, all possible attractors are placed in the above absorbing set. Fig. 4 shows the absorbing set and a self-excited attractor!
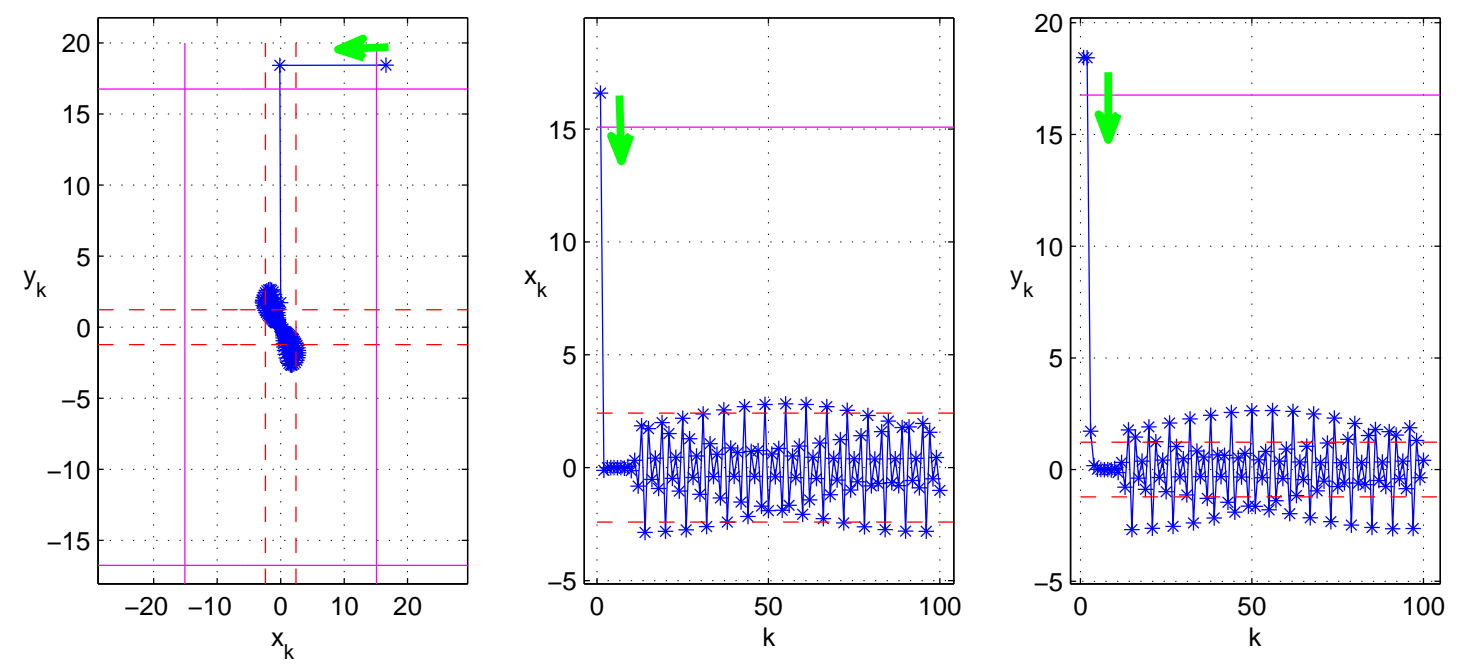

Figure 4: Case $3(|a|>1,|b|<1): \quad a=2.5, \quad b=0.1, \quad \delta=0.00001, \quad x_{1}=16.59, \quad y_{1}=18.44, \quad k=[1,100]$.

\footnotetext{
${ }^{1}$ An attractor is called a self-excited attractor if its basin of attraction intersects with any open neighborhood of an equilibrium; otherwise, it is called a hidden attractor [8, 10 12. Some recent examples of hidden attractors in continuous- and discrete-time dynamical systems can be found in [1, 2, 5, 7, 9,
} 


\section{Case 4: $|a|>1,|b|>1$. The existence of unbounded solutions} Then,

Consider a certain $\delta$ satisfying $|b|^{-1}<\delta<1$. Let $\left|y_{0}\right| \geq \sqrt{|a|-1}$ and $\left|x_{0}\right| \leq\left|y_{0}\right|\left(|b|-\delta^{-1}\right)$.

$$
\left|x_{1}\right|=\left|\frac{-a x_{0}}{1+y_{0}^{2}}\right| \leq\left|x_{0}\right|, \quad\left|y_{1}\right|=\left|b y_{0}+x_{0}\right| \geq\left|b y_{0}\right|-\left|x_{0}\right| \geq \delta^{-1}\left|y_{0}\right|>\left|y_{0}\right|,
$$

and $\left|x_{1}\right| \leq\left|y_{1}\right|\left(|b|-\delta^{-1}\right)$. Therefore,

$$
y_{k}\left(y_{0}\right) \underset{k \rightarrow \infty}{\longrightarrow} \infty
$$

Fig. 5 shows a solution, which tends to infinity.
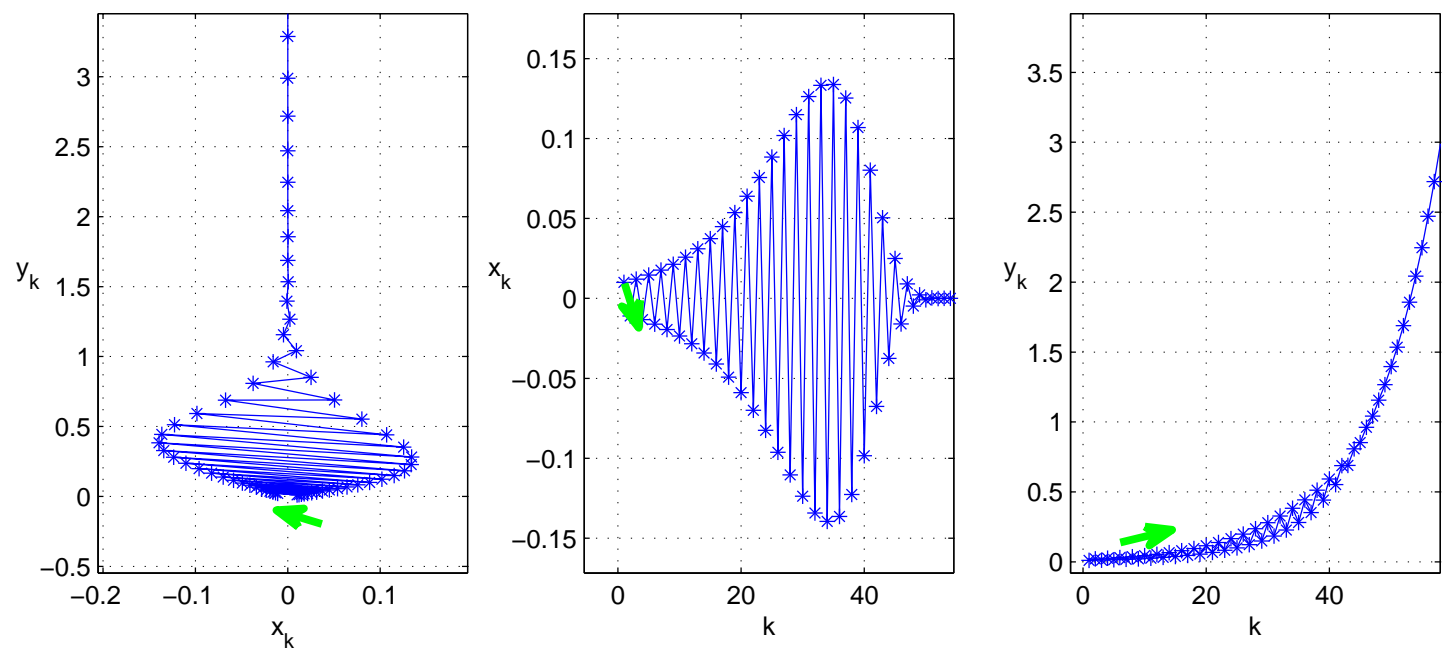

Figure 5: Case $4(|a|>1,|b|>1): \quad a=1.1, \quad b=1.1, \quad x_{1}=0.01, \quad y_{1}=0.01, \quad k=[1,60]$.

\section{Acknowledgment}

This work was supported by the Russian Scientific Foundation project 14-21-00041 and the Saint-Petersburg State University.

\section{References}

[1] Chen, G. [2015] "Chaotic systems with any number of equilibria and their hidden attractors," 4th IFAC Conference on Analysis and Control of Chaotic Systems (plenary lecture), http: //www.ee.cityu.edu.hk/ gchen/pdf/CHEN_IFAC2015.pdf.

[2] Danca, M.-F., Feckan, M., Kuznetsov, N. \& Chen, G. [2016] "Looking more closely to the Rabinovich-Fabrikant system," International Journal of Bifurcation and Chaos 26, 1650038.

[3] Zeraoulia, E. \& Sprott, J. [2011] "On the dynamics of a new simple 2-D rational discrete mapping," International Journal of Bifurcation and Chaos 21, 155-160.

[4] Zeraoulia, E. \& Sprott, J. [2011] "Some open problems in chaos theory and dynamics," International Journal of Open Problems in Computer Science and Mathematics 4, 1-10.

[5] Heath, W. P., Carrasco, J. \& de la Sen, M. [2015] "Second-order counterexamples to the discrete-time Kalman conjecture," Automatica 60, 140 - 144. 
[6] Jafari, S., Pham, V.-T., Golpayegani, S., Moghtadaei, M. \& Kingni, S. [2016] "The relationship between chaotic maps and some chaotic systems with hidden attractors," International Journal of Bifurcation and Chaos (Accepted).

[7] Kuznetsov, N. [2016] "Hidden attractors in fundamental problems and engineering models. A short survey." Lecture Notes in Electrical Engineering 371, 13-25, doi:10.1007/ 978-3-319-27247-4_2 (plenary lecture at AETA 2015: Recent Advances in Electrical Engineering and Related Sciences).

[8] Kuznetsov, N. V., Leonov, G. A. \& Vagaitsev, V. I. [2010] "Analytical-numerical method for attractor localization of generalized Chua's system," IFAC Proceedings Volumes (IFACPapersOnline) 4, 29-33, doi:10.3182/20100826-3-TR-4016.00009.

[9] Leonov, G., Kuznetsov, N. \& Mokaev, T. [2015] "Homoclinic orbits, and self-excited and hidden attractors in a Lorenz-like system describing convective fluid motion," European Physics Journal: Special Topics 224, 1421-1458, doi:10.1140/epjst/e2015-02470-3.

[10] Leonov, G. A. \& Kuznetsov, N. V. [2013] "Hidden attractors in dynamical systems. From hidden oscillations in Hilbert-Kolmogorov, Aizerman, and Kalman problems to hidden chaotic attractors in Chua circuits," International Journal of Bifurcation and Chaos 23, doi:10.1142/ S0218127413300024, art. no. 1330002.

[11] Leonov, G. A., Kuznetsov, N. V. \& Vagaitsev, V. I. [2011] "Localization of hidden Chua's attractors," Physics Letters A 375, 2230-2233, doi:10.1016/j.physleta.2011.04.037.

[12] Leonov, G. A., Kuznetsov, N. V. \& Vagaitsev, V. I. [2012] "Hidden attractor in smooth Chua systems," Physica D: Nonlinear Phenomena 241, 1482-1486, doi:10.1016/j.physd.2012.05. 016.

[13] Lu, J., Wu, X., Lu, J. \& Kang, L. [2004] "A new discrete chaotic system with rational fraction and its dynamical behaviors," Chaos, Solitons \& Fractals 22, 311 - 319.

[14] Zelinka, I. [2015] "A survey on evolutionary algorithms dynamics and its complexity - Mutual relations, past, present and future," Swarm and Evolutionary Computation 25, 2-14.

[15] Zelinka, I. [2016] "Evolutionary identification of hidden chaotic attractors," Engineering Applications of Artificial Intelligence 50, 159-167, 10.1016/j.engappai.2015.12.002. 